\title{
Optical thickness of galaxies
}

SIR - Burstein, Haynes and Faber ${ }^{1}$ claim to have demonstrated, for the first time, that spiral galaxies are optically thick at the faint isophote of 25th blue magnitude $\operatorname{arcsec}^{-2}\left(25 B_{n}\right)$. Apart from the astrophysical difficulties of such a dramatic hypothesis, which Burstein ef al. do not address, the claim itself is spurious because they neglect a wellknown selection effect which can nuturally explain all the trends in their 16 separate diagrams, even if all their galaxies are entirely transparent.

The test for opacity they are using is a minor variant of Holmberg's longstanding surface-brightness/inclination test ${ }^{2}$, to which the addition of distance information is largely irrelevant as both surface brightness and inclination are distant-independent quantities. What Burstein et al. have discovered in their data, as Valentijn ${ }^{3}$ did earlier, is that the measured surface brightnesses $\Sigma_{m} \equiv 1_{\text {ap }}$ $\pi a b$ of their galaxies are remarkably constant, that is, within $\pm 1.5 B_{u}$, a result made explicit in their Fig 6 .

There are two different interpretations of that result. Either the sample galaxies have been unconsiously selected to have a constant surface brightness, independent of inclination, in which case nothing whatsoever can be deduced about their opacity from the Holmberg test. Or the data are unaffected by such a selection effect, in which case their galaxies are indeed opaque at $25 B_{\mu}$ where the diameters were measured.

But it is well known ${ }^{3-5}$ that a sample of galaxies chosen for their apparent size, such as the UGC sample used by Burstein et al., is subject to very strong surface-brightness selection effects so that most objects in it, irrespective of their optical depths, will have measured $\Sigma_{m} \mathrm{~s}$ clustered within $\pm 1 \mathrm{mag}$ of a certain optimal value. This is because lower surface brightness galaxies hide so much of their light below the sky that they look small at a given isophote, whereas higher surface-brightness galaxies are, at a given luminosity, intrinsically and isophotally smaller. This striking effect, which acts independently of inclination ${ }^{6}$, renders Holmberg-type tests useless for determining optical depth.

It is up to Burstein et al. to retest their data and demonstrate that they are not explicable in this straightforward way. Readers of scientific papers can usually

1. Burstein, D. Haynes, M. \& Faber, S. Nature 353, 515-521 (1991)

. Holmberg. E Medd. Lund Ser II No 136 (1958)

3. Valentijn, E. Nature 346, $153-155$ (1990).

4. Disney, M. Nature 263, 573-575 (1976).

5. Disney, H. \& Phillipps, S. Mon. Not. R. astr. Soc. 205 , 1253-1265 (1983).

6. Davies, J. I. Mon. Not. R. astr. Soc. 244, 8-24 (1990)

7. Choloniewski, J. Mon. Not. R. astr. Soc. 250, 486-504 (1991). make such internal consistency checks for themselves. Unfortunately, although it purports to be a review, Burstein et al.'s paper is almost wholly based on what the authors themselves describe as a 'private database'. A paper based on 'hidden' data places itself above the normal processes of scrutiny that distinguish science from polemics.

Some results in science are harder to believe than they are to prove and the surprising effects of surface-brightness selection on galaxies may fall into this category. It is difficult, particularly for seasoned observers, to believe that they dominate almost all the discovered correlations between the global properties of galaxies, but they do.

MICHAEL DISNEY Department of Physics and Astronomy, University of Wales,

PO Box 913, Cardiff CF1 3TH, UK

BURSTEIN ET AL. REPLY - It is not sufficient to note, as Disney does, that two parameters (surface brightness and inclination) are distance-independent. We showed ${ }^{1}$ that the major factor in determining the results of various workers is sample selection, and sample selection has traditionally depended on the distant-dependent parameters of apparent magnitude and apparent diameter. Choloniewski has separately demonstrated this fact $^{7}$. Our method is fundamentally different from Holmberg's ${ }^{2}$ because we use the distance itself, in the form of the redshift, to break the former degeneracy between apparent and intrinsic quantities for galaxies. Indeed, our method cannot use surface brightness as part of the test.

If very low surface-brightness (VLSB) galaxies are excluded completely from these catalogues, our results naturally apply only to the higher surfacebrightness galaxies that are included. In this case, our result applies only to the given sample, which is all we have claimed. If, as Disney hypothesizes, VLSB galaxies are preferentially present as small-appearing galaxies in the ESO and UGC catalogues, they would affect our sample only if they had different inclination-dependent properties than those of higher surface-brightness galaxies. We agree that such an effect would preferentially affect the smaller appearing galaxies. That is one reason why we divide our sample into different ranges of apparent diameter. The fact that the result of inclination dependence of absolute diameter does not change with the apparent diameters of the galaxies would imply that significant contamination by VLSB galaxies has not affected our results for these higher surface-brightness galaxies.
Redshifts for most of the UGC galaxies used in our sample come primarily from published papers and catalogues (although our compilation of these data is private). ESO-LV data were used for our Fig. 6 (surface brightness versus inclination), as the edge-on galaxies in the UGC generally have unreliable magnitudes.

D. BURSTEIN

M. P. HAYNES

S. M. FABER

Department of Physics and Astronomy, Arizona State University,

Tempe, Arizona 85287-1504, USA

\section{No diabetes link to hsp65?}

SIR - Jones et al. ${ }^{1}$ suggest that the diabetes-susceptibility gene IDD5 may encode the mouse heat-shock protein 65 (hsp65), citing their previous report ${ }^{2}$ indicating that hsp65 is a $\beta$-cell antigen in insulin-dependent diabetes (IDD) in man.

Although such a link between hsp65 and IDD is attractive, the role of hsp65 as an autoantigen in IDD is by no means as generally accepted. Following the original report of Jones et $a l^{2}{ }^{2}$, two further reports ${ }^{3,4}$ indicated that the autoantigen in IDD was distinct from hsp65 on the basis of size, antibody reactivity and tissue distribution. Jones et al. subsequently indicated ${ }^{5}$ that the critical result in their original report may have been due to the presence in the IDD sera of antimycobacterial antibodies. Moreover, a well-documented paper ${ }^{6}$ identified the autoantigen in IDD as the $M_{\mathrm{r}} 64,000$ protein glutamic acid decarboxylase rather than hsp65.

Any assessment of the significance for diabetes of the similar chromosomal localization of IDD5 and an hsp65homologous gene must await good evidence that hsp65 is an autoantigen in human IDD. It may be of significance, however, that the Lsh/Ity/Bcg gene, which determines susceptibility to various infectious bacteria, also maps to this region of chromosome 1 (and is possibly identical to IDD5, ref. 7) as the main human immune response to these infectious agents is directed against their homologues of human hsp65 (ref. 8).

Department of Biochemistry, DAVID LATCHMAN

University College and Middlesex School of Medicine,

Cleveland St, London W1P 6DB, UK

1. Jones, D.B. et al. Nature 355, $119-120$ (1992).

2. Jones, D. B. et al. Nature 336, 583-585 (1990)

3. Kampe, O. et al. Lancet 336, 1250 (1990).

4. Atkinson, M.A. et al. Lancet 336, 1250-1251 (1990).

5. Jones, D.B. \& Duff, G. Lancet 337, 115 (1990)

6. Buckkeskov, S. et al. Nature 347, 151-156 (1990).

7. Cornall, R. J. et al. Nature 353, 262-265 (1991).

8. Kaufmann, S.H.E. Immun. Today 11, 129-136 (1990). 